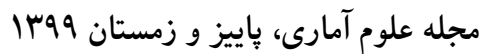

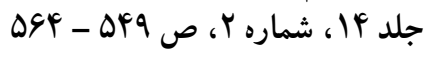 \\ DOI: $10.29252 /$ jss.14.2.549
}

\section{كلاسى از برآوردگرهاى انقباضى بيزى براى بارامتر مقياس توزيع وايبل براساس دادهاى

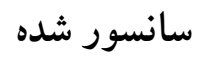

$$
\text { مهران نقىزاده قمى }
$$

كروه آمار، دانشگاه مازندران

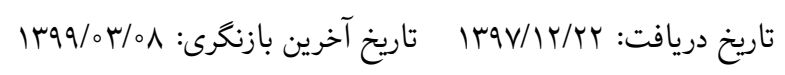

جكيده: در آمار كلاسيك، براساس اطلاعات نمونهاى و به كمك برآوردكرهاى معمول مانند برآوردكرهاى

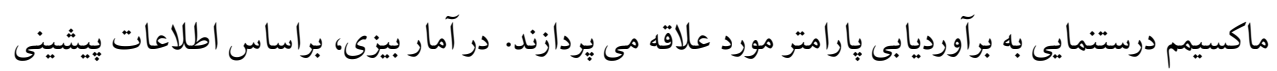

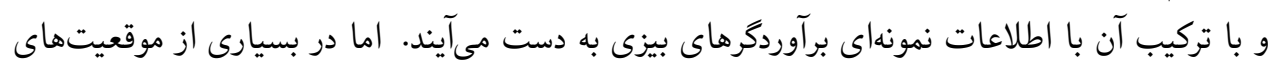

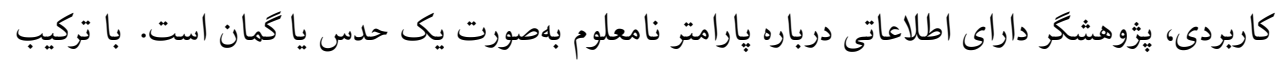

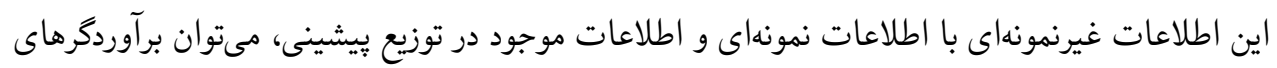

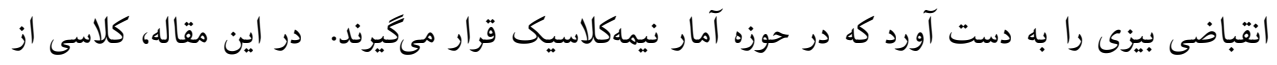

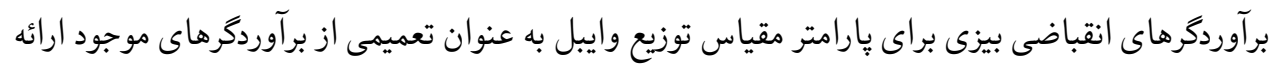

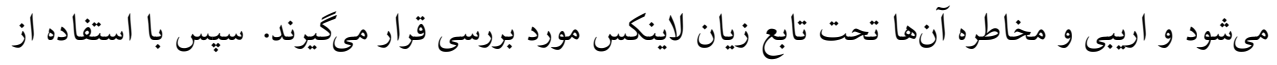

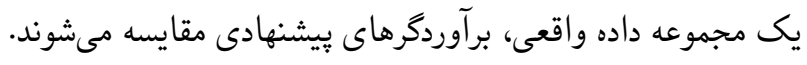
وازههاى كليدى: تابع زيان لاينكس، توزيع وايبل، برآوردگر انقباضى بيزى، دادهاى سانسورشده. 


\section{|}

فرض كنيد

$$
f(x ; \nu, \theta)=\frac{\nu}{\theta} x^{\nu-1} \exp \left(-\frac{x^{\nu}}{\theta}\right), \quad x>\circ, \quad \nu>, \quad \theta>\circ,
$$

باشد، كه در آن ل پارامتر شكل معلوم و $\theta$ يارامتر مقياس نامعلوم است. نمونهاى سانسورشده نوع دوم

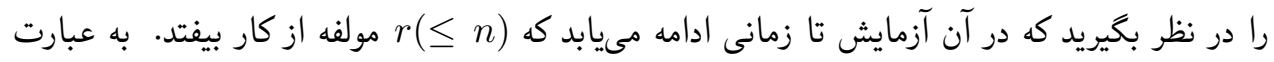

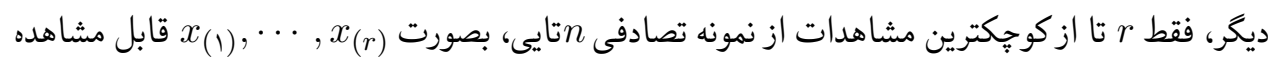

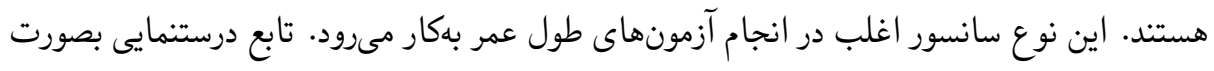

$$
L\left(\theta \mid x_{(1)}, \cdots, x_{(r)}\right)=\frac{\nu^{r}}{\theta^{r}} \Pi_{i=1}^{r} x_{i}^{\nu-1} \exp \left(-\frac{r \hat{\theta}}{\theta}\right),
$$

است، كه در آن ]

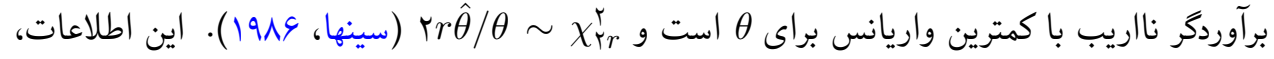

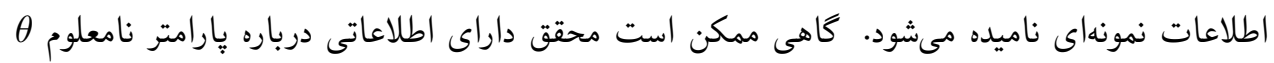

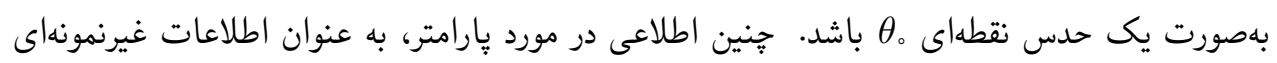

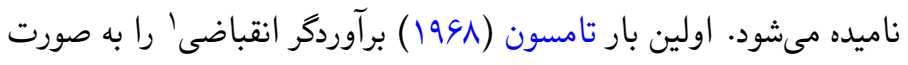

$$
S=k \hat{\theta}+(1-k) \theta_{\circ}, \quad \circ \leq k \leq 1,
$$

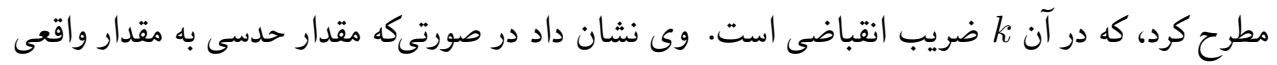

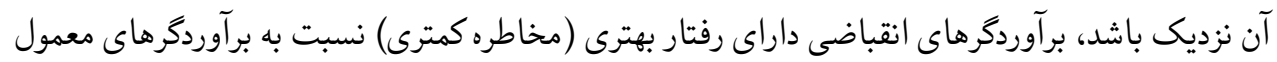

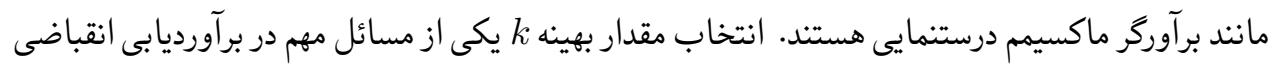

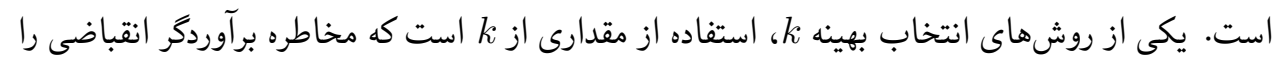

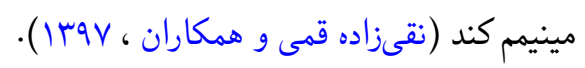

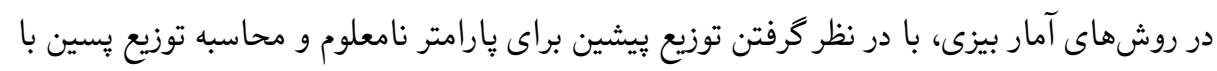


تركيب اطلاعات بيشينى و اطلاعات نمونهاى، برآوردكرهاى بيزى به دست مىآيند. درصورتىكه يك مقدار

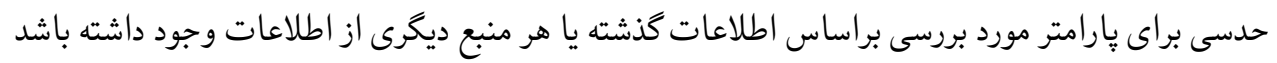

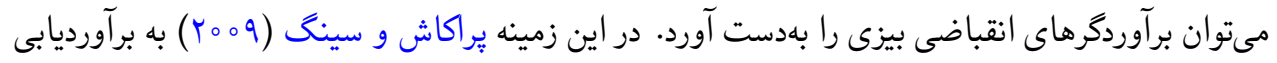

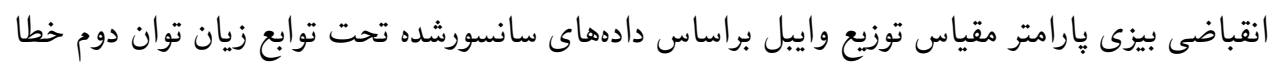

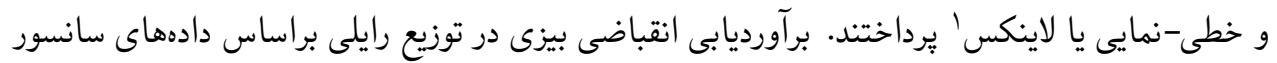

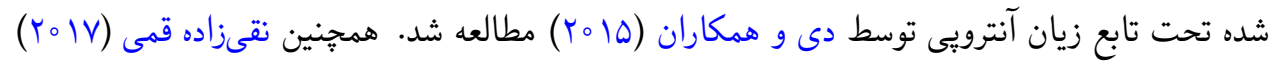

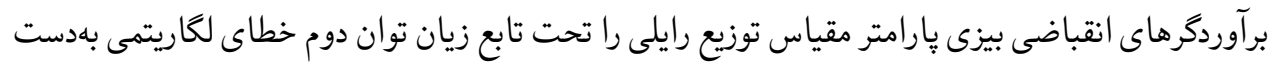

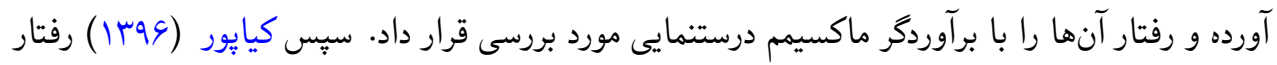

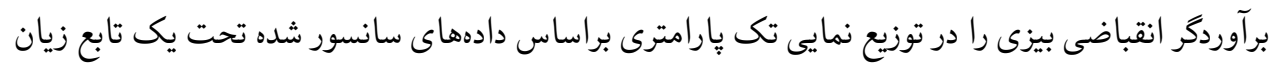
حزم انديشانه مورد مطالعه قرار داد.

در اين مقاله، كلاسى از برآوردكرهاى انقباضى بيزى براى يارامتر مقياس توزيع وايبل تحت تابع لاينكس

$$
L(\Delta)=\exp (a(\Delta-1))-a(\Delta-1)-1, a \neq \circ, \quad \Delta=\frac{\delta}{\theta}
$$

ارائه مىشود كه در آن $\delta$ يك برآوردكر دلخواه براى برآورد $\theta$ است. علامت و مقدار a بهترتيب جهت و

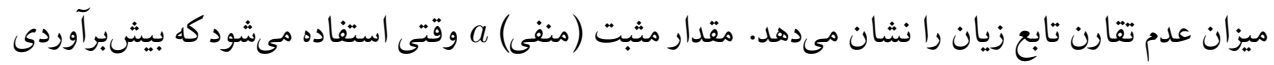

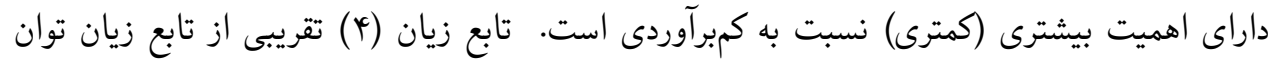

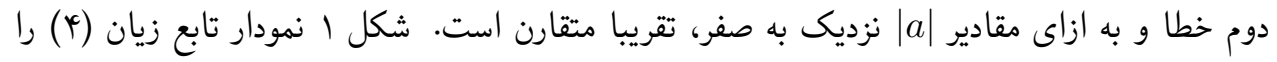

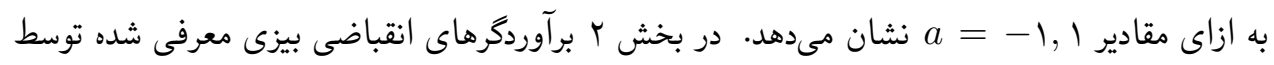

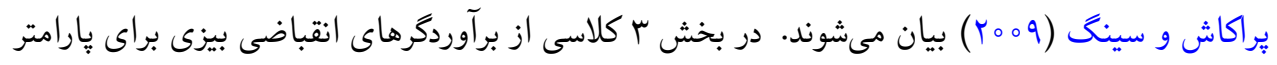

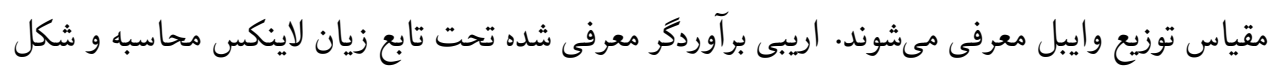

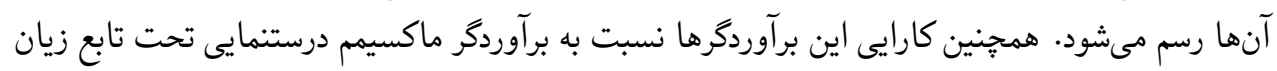

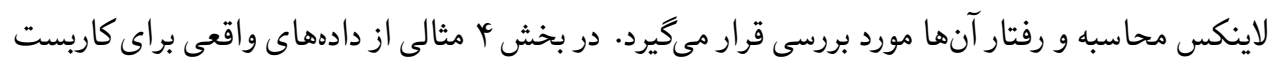

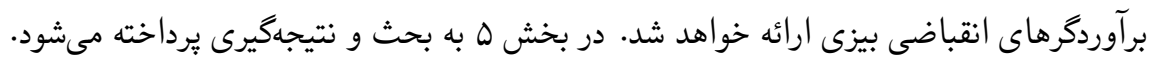




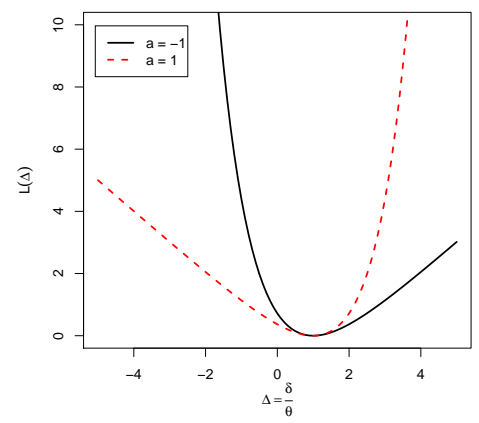

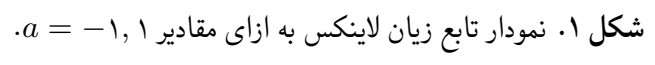

\section{r ب برآوردگ انقباضى بيزى}

$$
\begin{aligned}
& \text { با در نظر گرفتن توزيع يیشين گاماى وارونه براى } \theta \text { با تابع جگالى احتمال } \\
& \pi(\theta)=\frac{\beta^{\alpha} e^{-\frac{\beta}{\theta}}}{\Gamma(\alpha) \theta^{\alpha+1}}, \quad \alpha>\circ, \beta>\circ,
\end{aligned}
$$

$$
\begin{aligned}
& \text { و تركيب آن با تابع درستنمايى (Y)، توزيع يسين } \theta \text { به شرط (r) }
\end{aligned}
$$

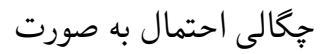

$$
\begin{aligned}
& \pi\left(\theta \mid \underline{X}^{r}\right)=\frac{(r \hat{\theta}+\beta)^{\alpha+r} e^{-\frac{r \hat{\theta}+\beta}{\theta}}}{\Gamma(\alpha+r) \theta^{\alpha+r+1}}, \alpha>\circ, \beta>\circ, \\
& \text { است. با مينيممردن مخاطره يسين به صورت } \\
& \mathrm{E}\left(L(\theta, \delta) \mid \underline{X}^{r}\right)=e^{-a} \mathrm{E}\left(e^{a \frac{\delta}{\theta}} \mid \underline{X}^{r}\right)-a \delta \mathrm{E}\left(\frac{1}{\theta} \mid \underline{X}^{r}\right)+a-1 \\
& \text { نسبت به ס، برآوردكر بيزى } \theta \text { تحت تابع زيان لاينكس بهصورت } \\
& \mathrm{E}\left(\frac{l}{\theta} e^{a \frac{\hat{\theta}_{B}}{\theta}} \mid \underline{X}^{r}\right)=e^{a} \mathrm{E}\left(\frac{l}{\theta} \mid \underline{X}^{r}\right)
\end{aligned}
$$


بهدست مىآيد. تبراشاش و سينگ (Y०9) نشان دادند برآوردكر بيزى $\theta$ برابر

$$
\hat{\theta}_{B}=\phi_{1}(r \hat{\theta}+\beta)
$$

است، كه در آن

$\phi_{1}=\frac{1}{a}\left(1-\phi_{r}^{-1}\right), \quad \phi_{r}=e^{\frac{a}{\alpha+r+1}}$.

با قرار دادن 。

$$
\beta=\theta_{\circ}\left(1-r \phi_{1}\right) \phi_{1}^{-1}
$$

به دست مىآيد. با قرار دادن مقدار م از (^) در (9) برآوردخر بيزى بهصورت

$$
\hat{\theta}_{B S}=r \phi_{1} \hat{\theta}+\left(1-r \phi_{1}\right) \theta_{\circ}=\lambda_{1} \hat{\theta}+\left(1-\lambda_{1}\right) \theta_{\circ}, \quad \lambda_{1}=r \phi_{1}
$$

حاصل مىشود، كه به صورت برآوردگر انقباضى (r) است و به اين دليل توسط يراكاش و سينگ (ro09)

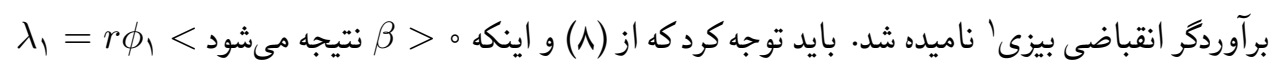

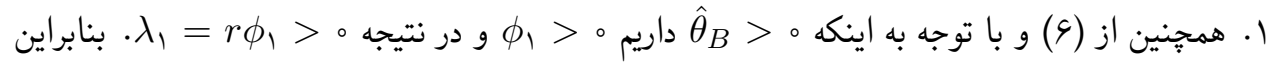

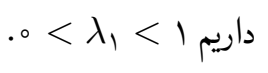

\section{r معرفى كلاس جديدى از برآوردگ هاى انقباضى بيزى}

در اين بخش كلاس جديدى از برآوردخرهاى انقباضى بيزى را معرفى كرده و به بررسى رفتار آن از نظر اريبى

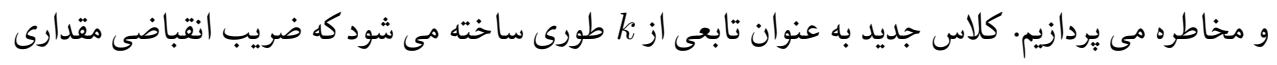
بين صفر و يك بوده و به ازاى ه 


$$
\begin{gathered}
\text { منظور، كلاس جديد از برآوردكرهاى انقباضى بيزى بهصورت } \hat{\theta}_{B S}^{(k)}=\frac{r\left(\phi_{r}-1\right)+\left[(a-r) \phi_{r}+r\right] k}{a \phi_{r}} \hat{\theta}+\frac{\left[(a-r) \phi_{r}+r\right](1-k)}{a \phi_{r}} \theta_{0} \\
=\lambda_{r} \hat{\theta}+\left(1-\lambda_{r}\right) \theta_{0}, \quad k \in[0,1] \\
\lambda_{r}=\frac{r\left(\phi_{r}-1\right)+\left[(a-r) \phi_{r}+r\right] k}{a \phi_{r}}
\end{gathered}
$$

مقدارى بين صفر و يك است كه در ادامه اثبات مى شود. به ازاى $\lambda_{r}=\frac{r\left(\phi_{r}-1\right)}{a \phi_{r}}=r \phi_{1}={ }^{\prime}=0$ , و در نتيجه

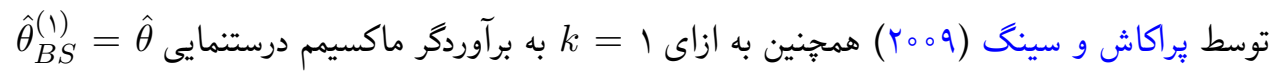

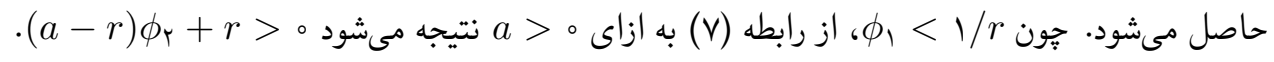

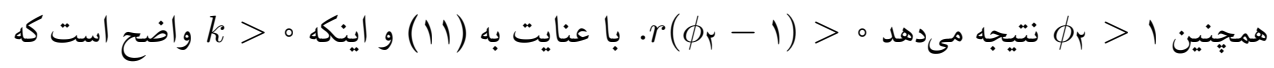

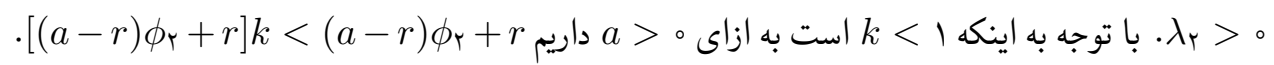
لذا

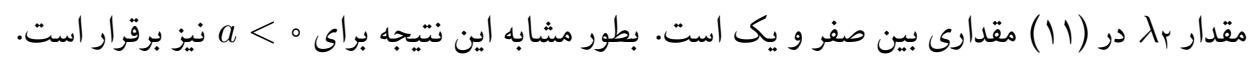

$$
\begin{aligned}
& \text { تعريف ا. (لهمن، 1901) برآوردكر } \delta \text { براى } 0 \text { مخاطره-نا/ ريب /ست آر } \\
& \mathrm{E}[L(\theta, \delta)] \leq \mathrm{E}\left[L\left(\theta^{\prime}, \delta\right)\right], \quad \theta \neq \theta^{\prime} .
\end{aligned}
$$

يافتن برآوردكرهاى مخاطره-ناريب به تابع زيان بستكى دارد. با توجه به تابع زيان لاينكس داريم

$$
\begin{aligned}
& \mathrm{E}[L(\theta, \delta)]-\mathrm{E}\left[L\left(\theta^{\prime}, \delta\right)\right]=\mathrm{E}\left[e^{a\left(\frac{\delta}{\theta}-1\right)}\right]-\mathrm{E}\left[e^{a\left(\frac{\delta}{\theta^{\prime}}-1\right)}\right]-a\left(\frac{1}{\theta}-\frac{1}{\theta^{\prime}}\right) \mathrm{E}[\delta] . \\
& \text { با قرار دادن }
\end{aligned}
$$$$
\mathrm{E}[L(\theta, \delta)]-\mathrm{E}\left[L\left(\theta^{\prime}, \delta\right)\right]=e^{-a}\left\{\mathrm{E}\left[e^{a \frac{\delta}{\theta}}-e^{a \frac{\delta}{\theta^{\prime}}}\right]-a e^{-a}\left(\frac{1}{\theta}-\frac{1}{\theta^{\prime}}\right) \mathrm{E}\left[\delta e^{a \frac{\delta}{\theta}}\right]\right\},
$$ 
با استفاده از نامساوى

$$
\mathrm{E}\left[e^{a \frac{\delta}{\theta}}-e^{a \frac{\delta}{\theta^{\prime}}}\right] \leq a\left(\frac{1}{\theta}-\frac{1}{\theta^{\prime}}\right) \mathrm{E}\left[\delta e^{a \frac{\delta}{\theta}}\right]
$$

بنابراين برآوردكر $\delta$ مخاطره-نااريب است اخر $\mathrm{E}[\delta]=e^{-a} \mathrm{E}\left[\delta e^{a \frac{\delta}{\theta}}\right.$ و اريبى برآوردكر $\delta$ بهصورت

$$
\operatorname{Bias}(\theta)=\mathrm{E}[\delta]-e^{-a} \mathrm{E}\left[\delta e^{a \frac{\delta}{\theta}}\right]
$$

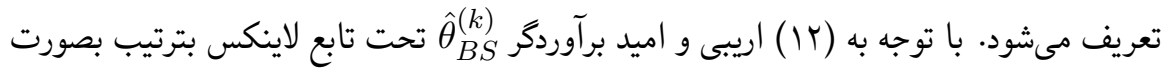

$$
\begin{aligned}
& \operatorname{Bias}(\theta)=\mathrm{E}\left[\hat{\theta}_{B S}^{(k)}\right]-e^{-a} \mathrm{E}\left[\hat{\theta}_{B S}^{(k)} e^{a \frac{\hat{\theta}_{B S}^{(k)}}{\theta}}\right], \\
& \mathrm{E}\left[\hat{\theta}_{B S}^{(k)}\right]=\theta\left[\lambda_{\digamma}+\left(1-\lambda_{\digamma}\right) \theta^{\star}\right] \\
& \text { به دست مىآيند، كه در آن } \\
& \mathrm{E}\left[\hat{\theta}_{B S}^{(k)} e^{a \frac{\hat{\theta}_{B S}^{(k)}}{\theta}}\right]=\theta \mathrm{E}\left[\left(\lambda_{\digamma} \frac{\hat{\theta}}{\theta}+\left(1-\lambda_{Y}\right) \theta^{\star}\right) e^{a\left(\lambda_{\digamma} \frac{\hat{\theta}}{\theta}+\left(1-\lambda_{\digamma}\right) \theta^{\star}\right)}\right] \\
& =\theta \mathrm{E}\left[\left(\frac{\lambda_{r}}{r r} U+\left(1-\lambda_{r}\right) \theta^{\star}\right) e^{a\left(\frac{\lambda_{r}}{r_{r}} U+\left(1-\lambda_{r}\right) \theta^{\star}\right)}\right] \\
& =\theta e^{a\left(1-\lambda_{r}\right) \theta^{\star}}\left\{\frac{\lambda_{r}}{r_{r}} \mathrm{E}\left[U e^{\frac{a \lambda_{r}}{r_{r}} U}\right]+\left(1-\lambda_{r}\right) \theta^{\star} \mathrm{E}\left[e^{\frac{a \lambda_{r}}{r r}} U\right]\right\},
\end{aligned}
$$

كه در آن U

$$
\begin{aligned}
\mathrm{E}\left[e^{\frac{a \lambda_{r}}{r r} U}\right]=\left(1-\frac{a \lambda_{r}}{r}\right)^{-r}, \quad \mathrm{E}\left[U e^{\frac{a \lambda_{r}}{r r} U}\right] & =\frac{r r^{r+r}}{\left(r+a \lambda_{r}\right)^{r+1}} . \\
& \text { در نتيجه اريبى برآوردكر } \hat{\theta}_{B S}^{(k)} \text { بصورت }
\end{aligned}
$$

$$
\begin{aligned}
\operatorname{Bias}(\theta) & =\theta\left\{\left(\lambda_{\digamma}+\left(1-\lambda_{\digamma}\right) \theta^{\star}\right)\right\} \\
& -\theta\left\{e^{-a} e^{a\left(1-\lambda_{\digamma}\right) \theta^{\star}}\left[\lambda_{\Gamma}\left(\frac{r}{\left(r+a \lambda_{Y}\right)}\right)^{r+1}+\left(1-\lambda_{\Upsilon}\right) \theta^{\star}\left(1-\frac{a \lambda_{\digamma}}{r}\right)^{-r}\right]\right\}
\end{aligned}
$$




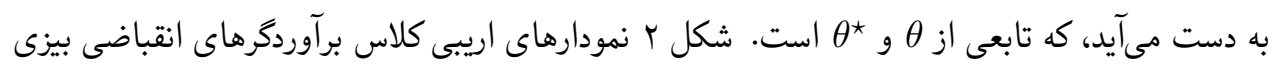

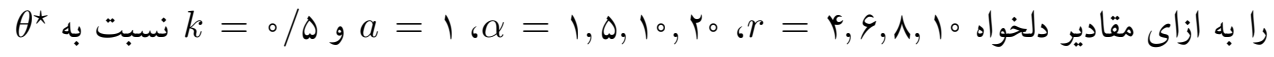

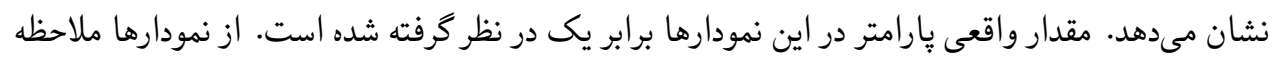

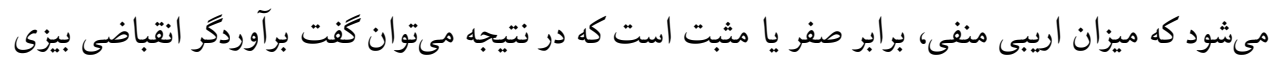

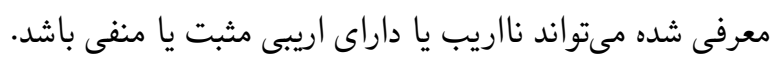

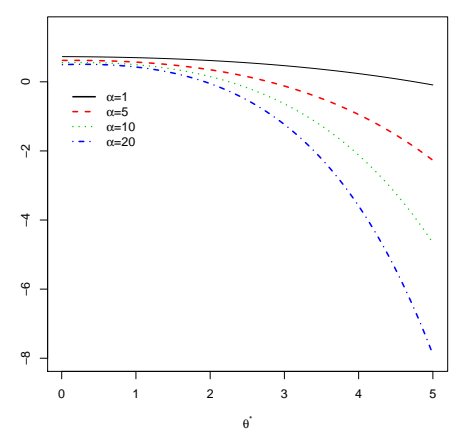

(ب)

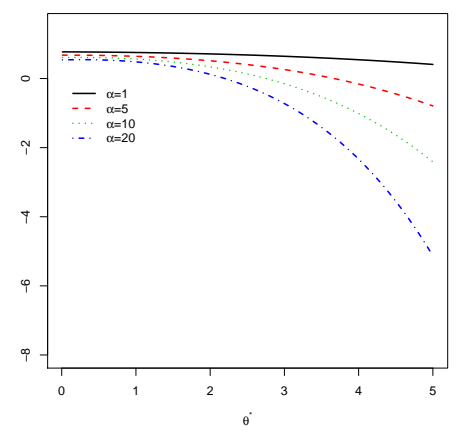

(د)

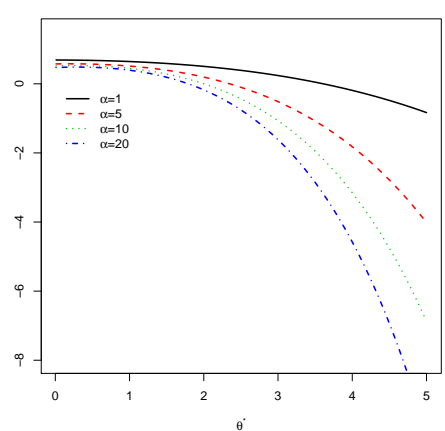

(الف)

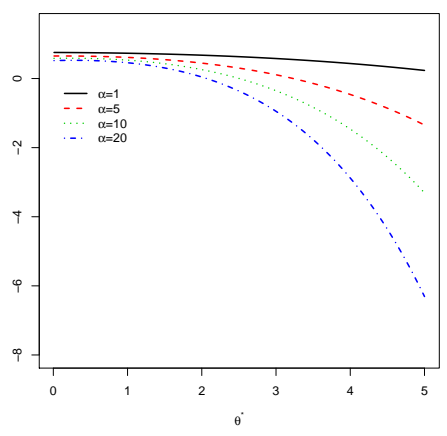

(ج)

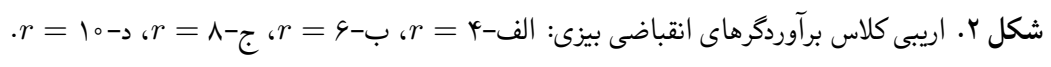




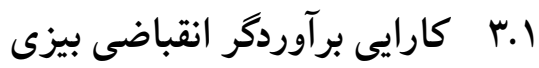

در اين بخش، كارايى برآوردكر انقباضى بيزى را نسبت به برآوردكر ماكسيمم درستنمايى مورد بررسى قرار

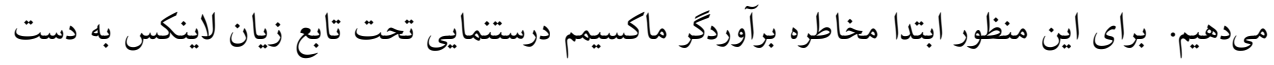

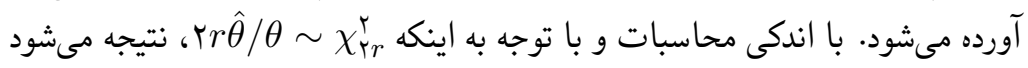

$$
R(\theta, \hat{\theta})=\mathrm{E}\left[e^{a\left(\frac{\hat{\theta}}{\theta}-1\right)}\right]-a \mathrm{E}\left[\frac{\hat{\theta}}{\theta}-1\right]-1=e^{-a}\left(\frac{r}{r-a}\right)^{r}-1 .
$$

اكنون مخاطره كلاس برآوردكرهاى انقباضى تحت تابع زيان لاينكس محاسبه مىشود، كه برابر است با

$$
\begin{array}{r}
R\left(\theta, \hat{\theta}_{B S}^{(k)}\right)=\mathrm{E}\left[e^{a\left(\frac{\hat{\theta}_{B S}^{(k)}}{\theta}-1\right)}\right]-a \mathrm{E}\left[\frac{\hat{\theta}_{B S}^{(k)}}{\theta}-1\right]-1 \\
\text { با توجه به رابطه (10) داريم }
\end{array}
$$

$$
\begin{aligned}
& \mathrm{E}\left[e^{a\left(\frac{\hat{\theta}_{B S}^{(k)}}{\theta}-1\right)}\right]=e^{a\left[\left(1-\lambda_{\digamma}\right) \theta^{\star}-1\right]}\left(1-\frac{a \lambda_{\digamma}}{r}\right)^{-r}, \\
& \mathrm{E}\left[\frac{\hat{\theta}_{B S}^{(k)}}{\theta}\right]=\left(1-\lambda_{\Upsilon}\right) \theta^{\star}+\lambda_{\digamma} .
\end{aligned}
$$

در نتيجه

$$
R\left(\theta, \hat{\theta}_{B S}^{(k)}\right)=e^{\left[\left(1-\lambda_{r}\right) \theta^{\star}-1\right]}\left(1-\frac{a \lambda_{r}}{r}\right)^{-r}+a\left(1-\lambda_{r}\right)\left(1-\theta^{\star}\right)-1
$$

حال براى بررسى رفتار كلاس برآوردگر انقباضى، كارايى نسبى آن را نسبت به برآورد ماكسيمم درستنمايى بلهورت

$R E\left(\hat{\theta}_{B S}^{(k)}, \hat{\theta}\right)=\frac{R(\theta, \hat{\theta})}{R\left(\theta, \hat{\theta}_{B S}^{(k)}\right)}=\frac{e^{-a}\left(\frac{r}{r-a}\right)^{r}-1}{e^{\left[\left(1-\lambda_{\digamma}\right) \theta^{\star}-1\right]}\left(1-\frac{a \lambda_{r}}{r}\right)^{-r}+a\left(1-\lambda_{\digamma}\right)\left(1-\theta^{\star}\right)-1}$

درنظر كرفته مىشود. شكل ץ نمودارهاى كارايى نسبى را به ازاى مقادير انتخابى

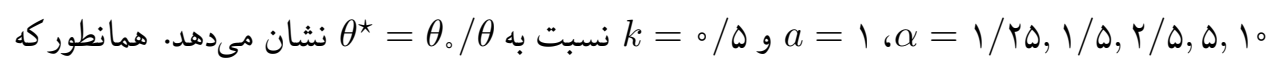


ملاحظه مىشود :

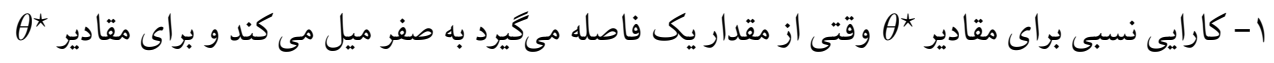

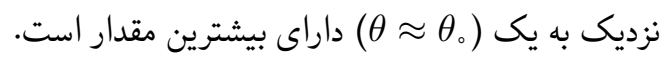

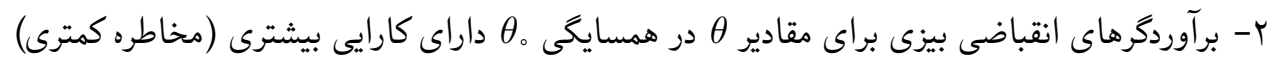

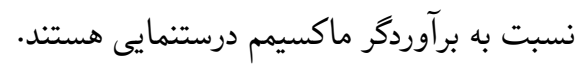

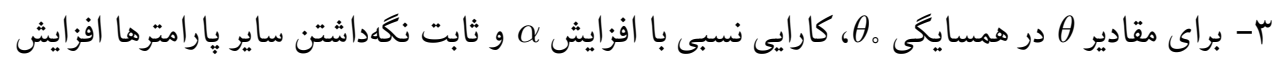

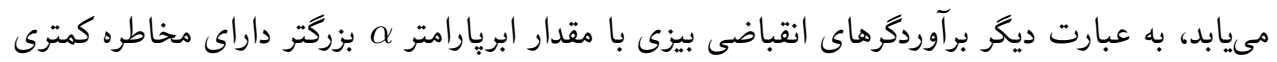

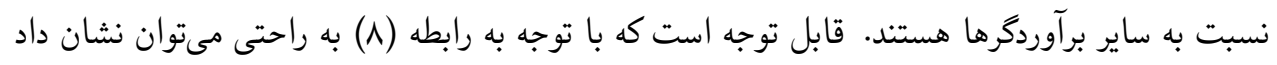

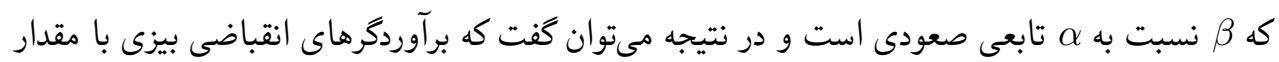

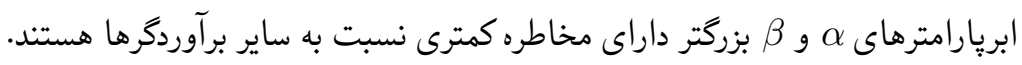

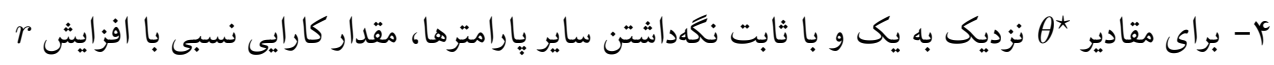
كاهش مىيابد. براى مقايسه برآوردخرهاى انقباضى بيزى در كلاس معرفى شده، شكل † ب رسم شده كه نمودار كارايى

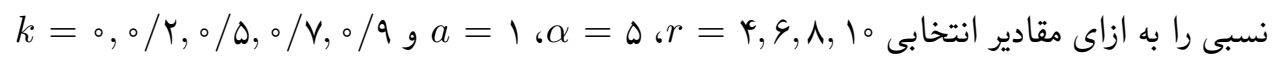

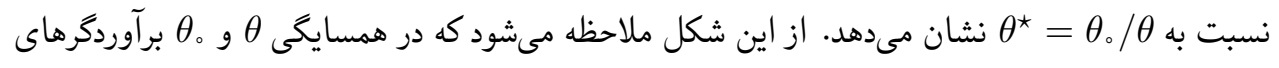

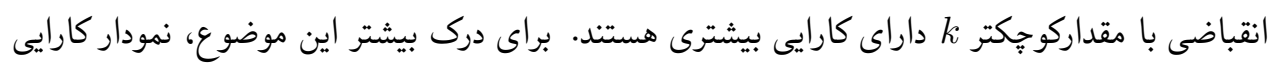

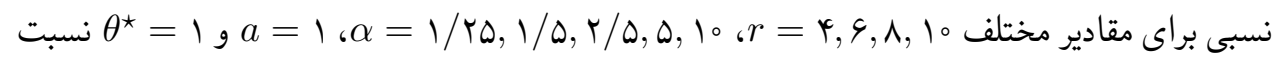

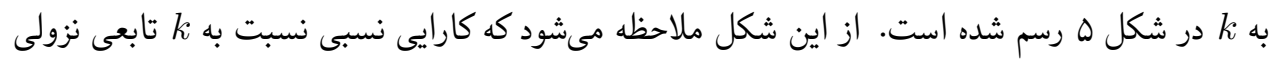
است به عبارت ديكر برآوردخرهاى انقباضى با مقدار ه

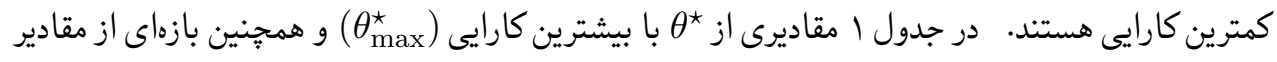

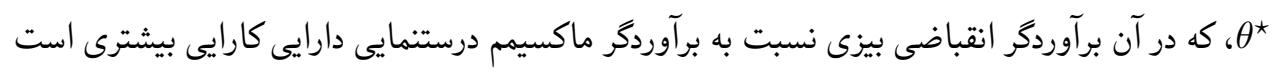

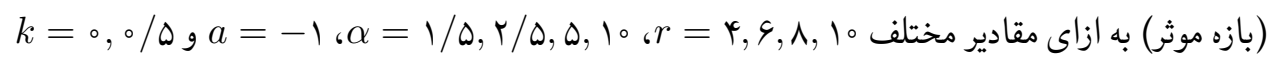

\section{p}

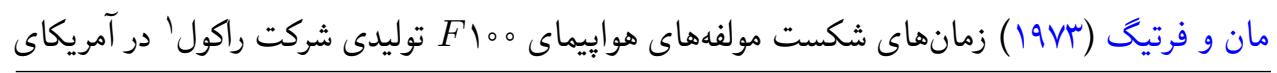




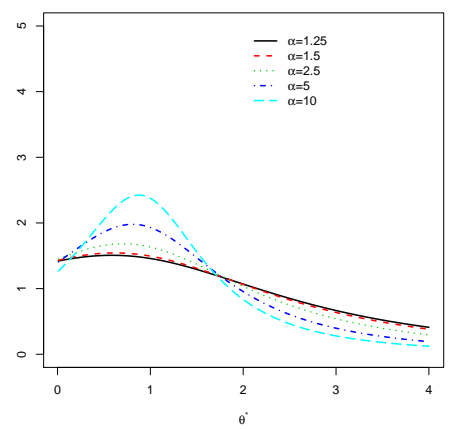

(ب)

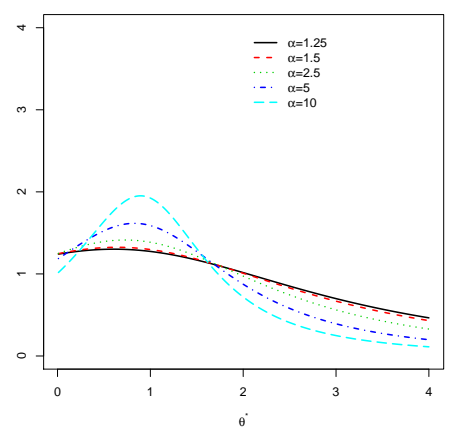

(2)

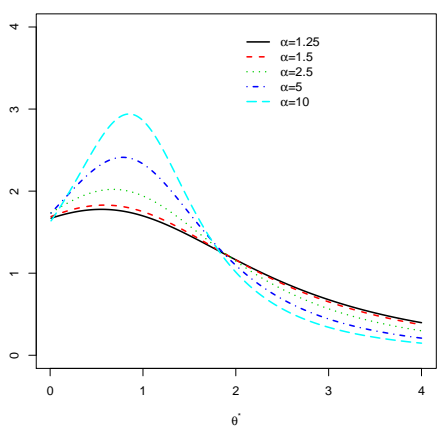

(الف)

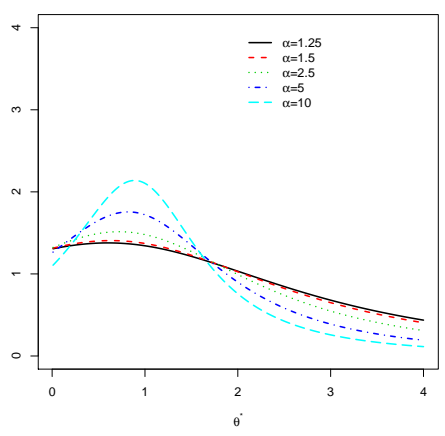

(ج)

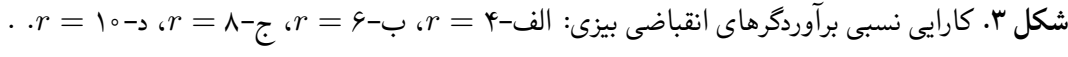

شمالى را مورد بررسى قرار دادند. با مولفه در آزمايش قرار كرفتند و زمانهاى شكست ما مولفه اول

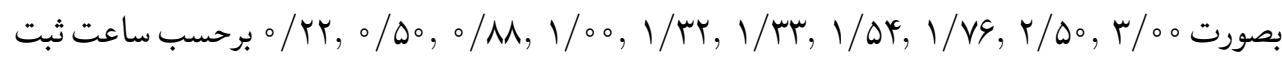

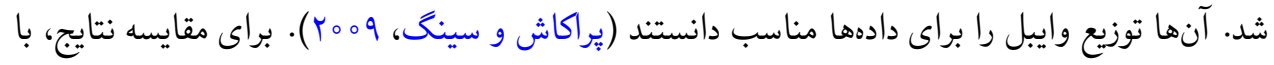

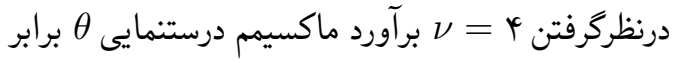
$\hat{\theta}=\frac{1}{10}\left[\sum_{i=1}^{10} x_{(i)}^{\kappa}+(\mid r-10) x_{(10)}^{\kappa}\right]=r \Lambda / 411 \%$.

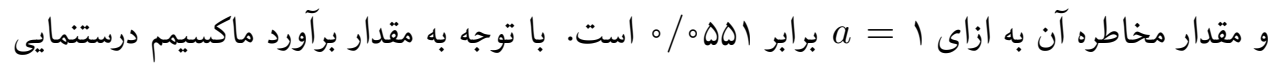




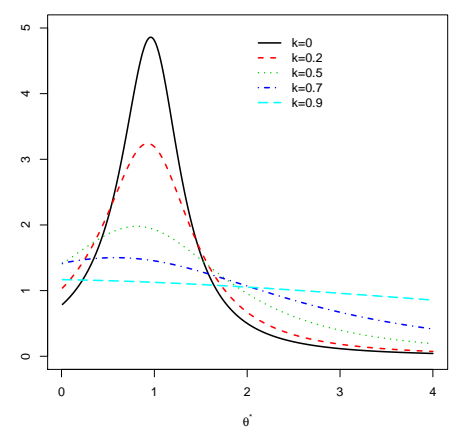

(ب)

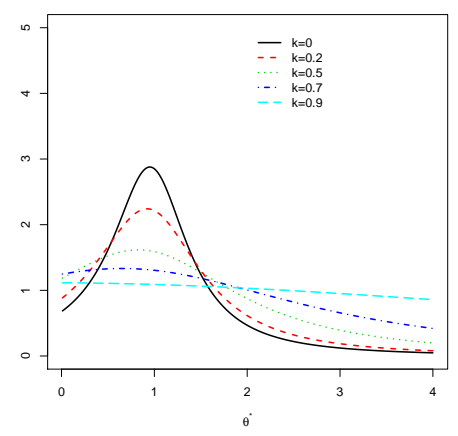

(د)

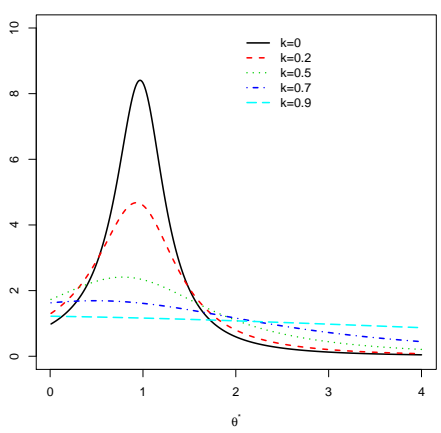

(الف)

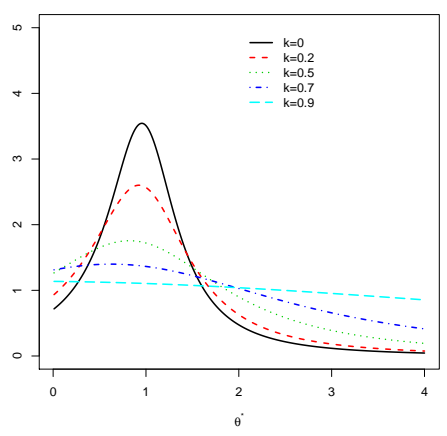

(ج)

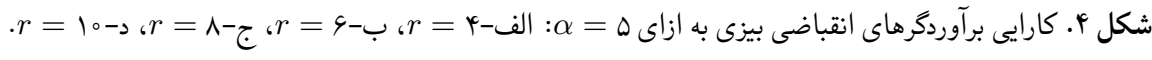

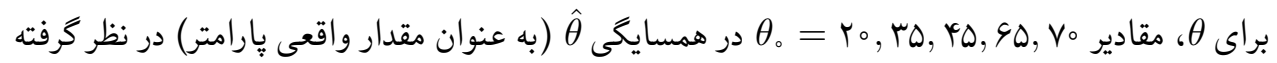

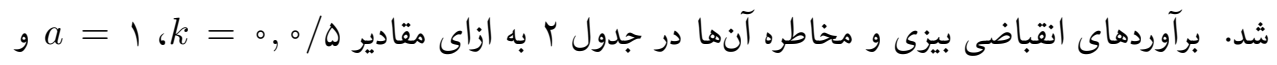
حداس

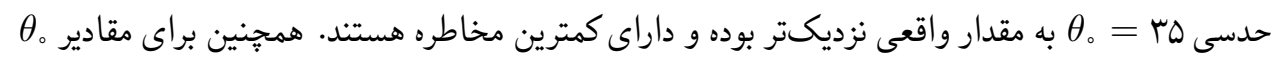

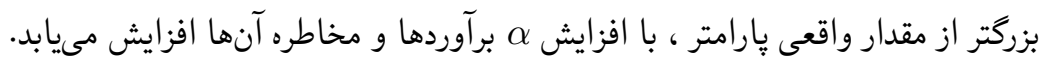




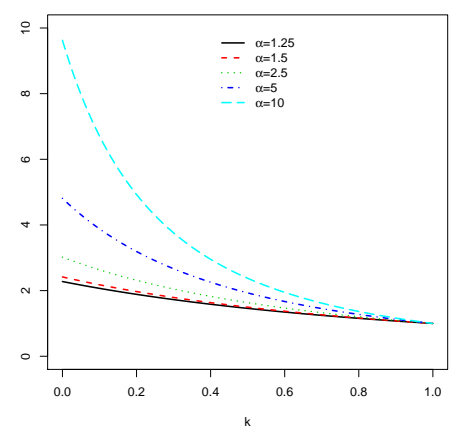

(ب)

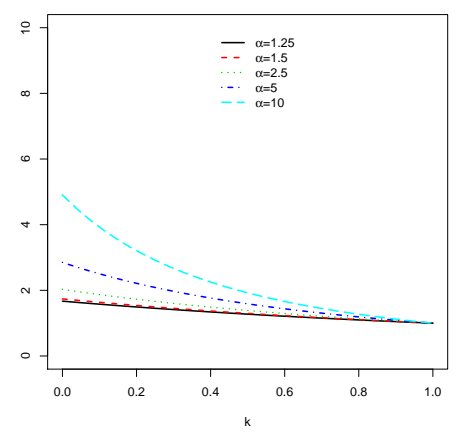

(د)

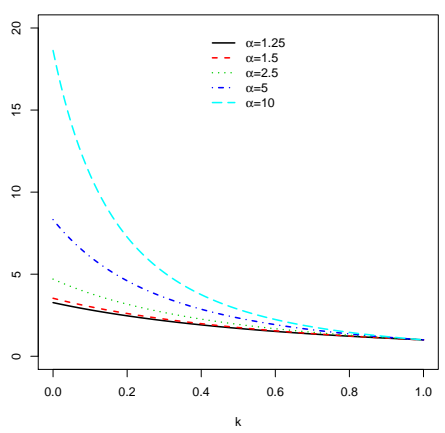

(الف)

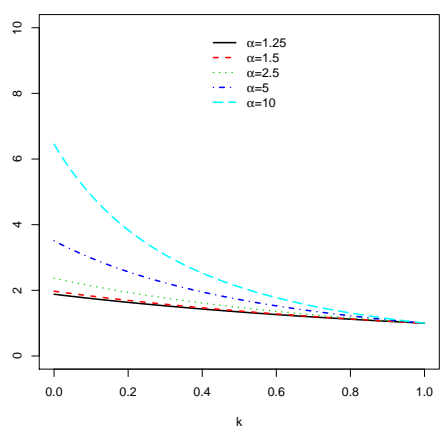

(ج)

شكل ه. كارايى برآوردكرهاى انقباضى بيزى به ازاى أ =

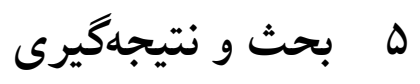

در اين مقاله، يك كلاس از برآوردگرهاى انقباضى بيزى براى بارامتر مقياس توزيع وايبل براساس دادههاى

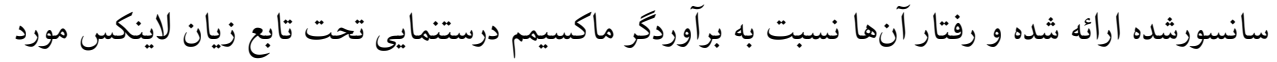

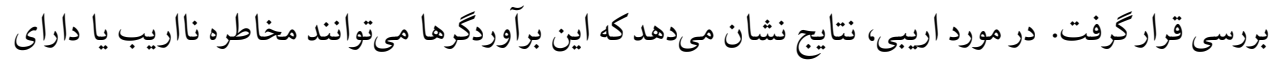

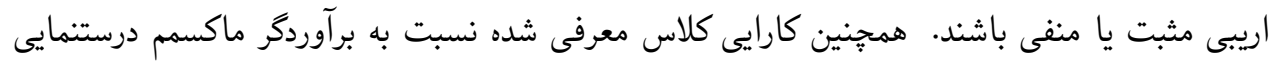

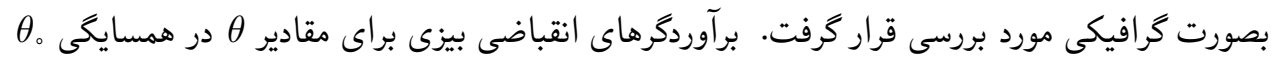

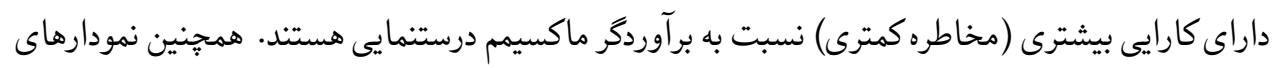


. . . كلاسى از برآوردكرهاى انقباضى بيزى

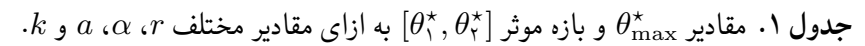

\begin{tabular}{|c|c|c|c|c|c|}
\hline \multicolumn{2}{|c|}{$\alpha=r / \Delta$} & \multicolumn{2}{|l|}{$\alpha=1 / \Delta$} & \multirow[b]{2}{*}{$k$} & \multirow[b]{2}{*}{$r$} \\
\hline$\left[\theta_{1}^{\star}, \theta_{\Gamma}^{\star}\right]$ & $\theta_{\max }^{\star}$ & {$\left[\theta_{1}^{\star}, \theta_{\Upsilon}^{\star}\right]$} & $\theta_{\max }^{\star}$ & & \\
\hline $\begin{array}{c}{[\circ / T H Y q, Y / \circ \Delta \Lambda \Lambda]} \\
{[\circ, \quad Y / V q \circ V]}\end{array}$ & $\begin{array}{l}1 / 0 \wedge 90 \\
1 / 419 V\end{array}$ & $\begin{array}{c}{[0 /|| r r g, r / r \wedge \|]} \\
{[0, r /|q| \Lambda]}\end{array}$ & $\begin{array}{l}1 / 14 \wedge q \\
1 / 44 \Delta \Delta\end{array}$ & $\begin{array}{l}\circ \\
\circ / 0\end{array}$ & r \\
\hline $\begin{array}{l}{[0 / Y \varphi \circ G, Y / \circ M Y Y]} \\
{[0 / \circ Y \Delta Q, Y / Y \circ \wedge Y]}\end{array}$ & $\begin{array}{l}1 / 10 \mu 1 \\
1 / 4 \mid v 1\end{array}$ & $\begin{array}{c}{[0 / 1901, Y / T V I V]} \\
{[0, r /|9| \Lambda]}\end{array}$ & $\begin{array}{l}1 /|V Y| \\
1 / \text { YGTY }\end{array}$ & $\begin{array}{l}\circ \\
\circ / 0\end{array}$ & 9 \\
\hline $\begin{array}{l}{[\circ / Y V G Y, Y / \circ Y \circ G]} \\
{[\circ / \circ Y \wedge V, Y / G G \Delta V]}\end{array}$ & $\begin{array}{l}1 / 11 r q \\
1 / \pi 1 \wedge r\end{array}$ & $\begin{array}{c}{[0 / I V V \circ, r / Y \& q Y]} \\
{[\circ, r / \circ V V \Lambda]}\end{array}$ & $\begin{array}{l}1 / 1194 \\
1 / 4994\end{array}$ & $\begin{array}{l}\circ \\
\circ / 0\end{array}$ & $\wedge$ \\
\hline $\begin{array}{l}{[0 / Y \wedge \& \Delta, Y / 0 \mid K Y]} \\
{[0 / 0 q K Y, Y / G T q \Lambda]}\end{array}$ & $\begin{array}{l}1 / 4119 \\
1 / 419 V\end{array}$ & $\begin{array}{c}{[0 / \mid \wedge Y q, r / r \& q 1]} \\
{[0, r / 09 \mid r]}\end{array}$ & $\begin{array}{l}1 / 1999 \\
1 / 4 V Y V\end{array}$ & $\begin{array}{l}\circ \\
\circ / 0 \\
\end{array}$ & 10 \\
\hline \multicolumn{2}{|l|}{$\alpha=1 \circ$} & \multicolumn{2}{|l|}{$\alpha=\emptyset$} & & \\
\hline $\begin{array}{l}{[0 / K Y G Y, 1 / 9901]} \\
{[0 / \mid Y V Y, Y / Y 09 \Lambda]}\end{array}$ & $\begin{array}{l}1 / 01 r \Delta \\
1 / r|V|\end{array}$ & 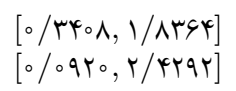 & $\begin{array}{l}1 / \circ r \Delta V \\
1 / 1949\end{array}$ & $\begin{array}{l}\circ \\
\circ / 0\end{array}$ & i \\
\hline 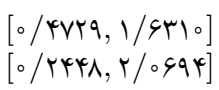 & $\begin{array}{l}1 / 0199 \\
1 / 114 r\end{array}$ & $\begin{array}{l}{[0 / r V q r, \mid / V \wedge q r]} \\
{[0 /|⿱ \& q 1, r / r| q r]}\end{array}$ & $\begin{array}{l}1 / 044 Y \\
1 / 1109\end{array}$ & $\begin{array}{l}\circ \\
\circ / 0\end{array}$ & 4 \\
\hline $\begin{array}{l}{[0 / \Delta 000,1 / \Delta 9 G T]} \\
{[\circ / T \wedge \Lambda \Delta, 1 / 99 \mu T]}\end{array}$ & $\begin{array}{l}1 / 0191 \\
1 / 1094\end{array}$ & $\begin{array}{l}{[\circ / Y \circ \circ Y, Y / V G \Delta r]} \\
{[\circ / Y \wedge Y T, Y / Y \Delta \Delta r]}\end{array}$ & $\begin{array}{l}\text { I/OOTK } \\
\text { I/TH }\end{array}$ & $\begin{array}{l}\circ \\
\circ / 0\end{array}$ & $\wedge$ \\
\hline $\begin{array}{l}{[0 / \Delta I V \Lambda, 1 / \Delta V \psi V]} \\
{[0 / Y I V V, 1 / q K \psi k]}\end{array}$ & $\begin{array}{l}\text { VOTYY } \\
1 / 10 Y 9\end{array}$ & $\begin{array}{l}{[\circ / Y|Y|, \mid / V \Delta \circ q]} \\
{[\circ / r \circ r q, r / r \mid V Y]}\end{array}$ & $\begin{array}{l}1 / 0091 \\
1 / 1109\end{array}$ & $\begin{array}{l}\circ \\
\circ / 0 \\
\end{array}$ & 10 \\
\hline
\end{tabular}

\begin{tabular}{|c|c|c|c|c|c|}
\hline \multicolumn{4}{|c|}{$\alpha$} & \multirow[b]{2}{*}{$k$} & \multirow[b]{2}{*}{$\theta$} \\
\hline$r_{0}$ & 10 & 0 & $r / \Delta$ & & \\
\hline 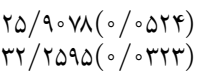 & 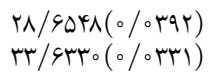 & $\begin{array}{l}r Y / r V \Delta q(0 / \% r T q) \\
r Y / q K r \Delta(\% / \% \Delta Q q)\end{array}$ & $\begin{array}{l}r r / r \Lambda V \Lambda(0 / \% r T V) \\
r \Delta / q Y q \Delta(0 \% \text { \% } q Y)\end{array}$ & $\circ$ & $r_{0}$ \\
\hline $\begin{array}{l}r q / \operatorname{rgq}(0 \% \circ 9 \Lambda) \\
r V / \text { rVAV }(0 \% \text { TrV })\end{array}$ & 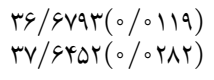 & $\begin{array}{l}r V / \Lambda \Lambda V q(0 / 019 T) \\
r V / \wedge 99 \Delta(0 \% \text { HYr })\end{array}$ & $\begin{array}{l}r v / \Delta V \Lambda r(0 / 0 r q \Lambda) \\
r \Lambda / \circ q r v(0 \% \text { O } q r)\end{array}$ & $\circ / 0$ & ro \\
\hline 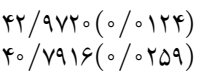 & 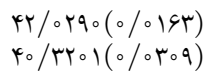 & 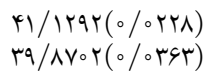 & 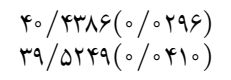 & $\stackrel{\circ}{\circ / 0}$ & is \\
\hline $\begin{array}{l}\Delta r / r \mid \circ \Delta(0 /|r q|) \\
\psi \Delta / 910 \Lambda(0 / 0 \Delta \Lambda Y)\end{array}$ & 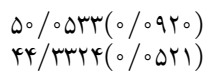 & $\begin{array}{l}\text { YV/OFIT }(0 / .900) \\
\text { YY/ATGT }(0 / 0491)\end{array}$ & $\begin{array}{l}r Y / V Y q 0 /(0 / 0 \Delta r Y) \\
Y Y / q V 01(0 / 0 \psi \Delta V Y)\end{array}$ & $\circ$ & 90 \\
\hline $\begin{array}{l}90 / . r 41(r / 1901) \\
r 9 / r r T V(0 / 0 V Y q)\end{array}$ & 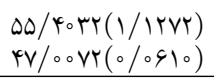 & $\begin{array}{l}\Delta \circ / q \Lambda T \Delta(0 / 0 \Delta r q) \\
K Y / V Q 9 \Lambda(0 / 0 \Delta K r)\end{array}$ & $\begin{array}{l}q V / \Delta \Lambda q T(0 / 0 q T \Delta) \\
K r / 100 Y(0 / 0 \Delta I V)\end{array}$ & $\begin{array}{l}\circ \\
\circ / 0 \\
\end{array}$ & $v_{0}$ \\
\hline
\end{tabular}


كارايى نسبى نسبت به k رسم شدند كه نشان مىدهد برآوردگرهاى انقباضى با مقداركوجكتر k داراى كاراى

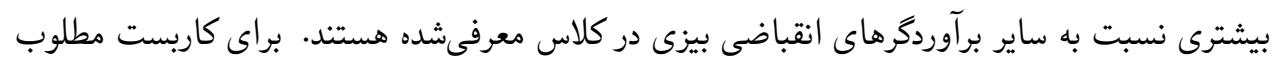

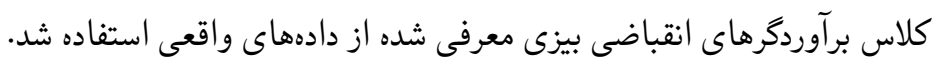

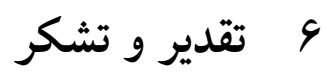

نويسنده، از سردبير، داوران و ويراستار محترم مجله براى ارزيابى و بهبود كيفيت مقاله كمال تشكر را دارد.

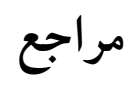

كيايور، آ. (49+1)، برآوردگ انقباضى بيزى براى يارامتر مقياس توزيع نمايى براساس دادهاى سانسورشده،

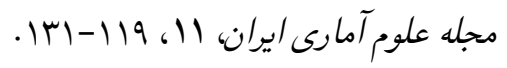

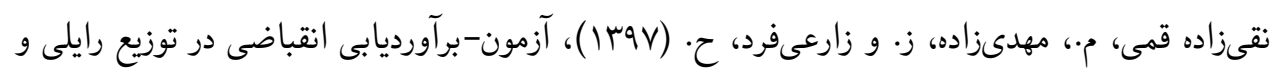

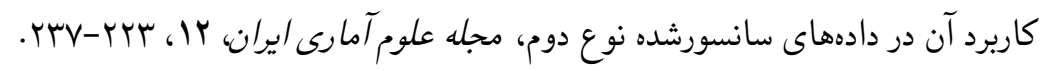

Dey, Sanku., Dey, T. and Maiti, S. (2015), Bayes Shrinkage Estimation of the Parameter of Rayleigh Distribution for Progressive Type-II Censored Data, Austrian Journal of Statistics, 44, 3-15.

Lehmann, E. L. (1951), A General Concept of Unbiasedness, The Annals of Mathematical Statistics, 22, 587-592.

Mann, N. R. and Fertig, K.W. (1973), Tables for Obtaining Confidence Bounds and Tolerance Bounds Based on Best Linear Invariant Estimates of Parameters of the Extreme Value Distribution, Technometrics, 15, 87101.

Naghizadeh Qomi, M. (2017), Bayesian Shrinkage Estimation Based on Rayleigh Type-II Censored Data, Communications in Statistics-Theory and Methods, 46, 9859-9868. 
Prakash, G., and Singh, D. C. (2009), A Bayesian Shrinkage Approach in Weibull Type-II Censored Data Using Prior Point Information, REVSTAT-Statistical Journal, 7, 171-187.

Sinha, S.K. (1986), Reliability and Life-Testing, Wiley Eastern, New Delhi.

Thompson, J. R. (1968), Some Shrunken Techniques for Estimating the Mean, Journal of the American Statistical Association, 63, 113-122. 
Journal of Statistical Sciences, Autumn and Winter, 2020

Vol. 14, No. 2, pp 549-564

DOI: $10.29252 /$ jss.13.2.549

\title{
A Class of Bayesian Shrinkage Estimators for the Scale Pa- rameter of Weibull Distribution Based on Censored Data
}

Naghizadeh Qomi, M.

Departmet of Statistics, University of Mazandaran, Babolsar, Iran.

\begin{abstract}
In classical statistics, the parameter of interest is estimated based on sample information and using natural estimators such as maximum likelihood estimators. In Bayesian statistics, the Bayesian estimators are constructed based on prior knowledge and combining with it sample information. But, in some situations, the researcher has information about the unknown parameter as a guess. Bayesian shrinkage estimators can be constructed by Combining this non-sample information with sample information together with the prior knowledge, which is in the area of semi-classical statistics. In this paper, we introduce a class of Bayesian shrinkage estimators for the Weibull scale parameter as a generalization of the estimator at hand and consider the bias and risk of them under LINEX loss function. Then, the proposed estimators are compared using a real data set.
\end{abstract}

Keywords: LINEX loss function, Weibull distribution, Bayesian Shrinkage testimator, Censored data.

Mathematics Subject Classification (2010): 62F10, 62 F15. 